\title{
State-Dependence and Conditional Audit Policy
}

\author{
Ruey-Ji Guo, Yenpao Chen, Chun Chen Lee \\ Department of Accounting, Soochow University, Taipei, Taiwan \\ Email: grj@scu.edu.tw, yenpao@scu.edu.tw, cclee@scu.edu.tw
}

Received July 30, 2013; revised August 30, 2013; accepted September 9, 2013

Copyright (C) 2013 Ruey-Ji Guo et al. This is an open access article distributed under the Creative Commons Attribution License, which permits unrestricted use, distribution, and reproduction in any medium, provided the original work is properly cited.

\begin{abstract}
This paper investigates how the state-dependence between two periods affects the optimal audit decision. Using a principal-agent model, based on a state-dependent assumption, we find that it is desirable to use a conditional (punitive) audit mechanism when the agent's under-declaration benefit is significant and the principal's audit cost is moderate. In that case, the audit policy for the current period will be contingent on the audit result in the preceding period.
\end{abstract}

Keywords: Information Asymmetry; Agency Problem; Audit Policy

\section{Introduction}

To alleviate the problem resulting from information asymmetry, the principal tends to use audit mechanism to abate the related agency costs, or to maximize the net revenues concerned. The past studies have paid considerable attention to the audit issues, including Antle [1], Baron and Besanko [2], Demski and Sappington [3], and Baiman, Evans and Noel [4]. Variables of particular interest are the audit probability and the conditions upon which it depends. Using the data from the American pulp and paper industry, Helland [5] found that firms which are discovered in violation of environmental regulations experience a one- or two-quarter period of more frequent inspections from regulators. Placing higher inspection probability on agents with the record of incompliance appears to be a scheme preferred by the regulators.

Audit rules conditional on compliance history are broadly adopted by researchers as well. For instance, Landsberger and Meilijson [6] proposed that an agent's current audit results affect its probability of future audits. By targeting audits based on current audit outcomes, They demonstrated that conditional auditing is more cost-effective than random auditing and that agencies could increase tax revenue given the same enforcement budget and fine scheme. The use of such endogenous and dynamic enforcement mechanisms that use information obtained through prior audit to assign the agent's probability of future audits are extended to the literature of pollution regulation and tax compliance (e.g. Greenberg [7], Harrington [8], Friesen [9], and Stafford [10]).

In this paper, we present a two-period model with a state-dependent scenario, i.e. the nature state in period two is correlated with that in period one, to address the principal's audit decision and study the potential effect of state-dependence between different periods. Essentially, audit mechanism is associated with penalty system. As Landsberger and Meilijson [6] note that penalty systems usually include two elements of both penalty function and probability of detection, while from the practical point of view, the latter more than the former is under the control of the authorities. Accordingly, this paper focuses on the issue of optimal audit probability (probability of detection), and aims to identify the desirability of conditional audit.

By controlling the state-dependent degree of return, we demonstrate that a conditional audit policy can make the audit implementation more efficient only when the auditee's benefit of under-declaring return is larger than the expected penalty under complete audit and the audit cost is relatively moderate. In that case, by using conditional audit mechanism, the principal can concentrate costly audit resources on the auditees with higher probability to under-declare their actual returns. Additionally, we find that the state-dependent degree of return indeed plays a prominent role in whether to use conditional audit policy or not. This paper points out it may not be desirable for the principal to implement any conditional audit especially in a situation that the returns in two periods are totally independent.

\section{The Model}

In this paper, we use a principal-agent hierarchy, including a principal, an auditor and a manager, to address the issue of conditional audit in a two-period scenario. It is 
assumed that, in a vertical structure owned by the principal, the manager operates a business unit and holds private information concerning its realized returns. The principal can obtain the true return information only by assigning the auditor to undertake audit task. For sim= plicity, it is assumed that all parties are risk neutral, and the auditor is absolutely independent.

In the two-period scenario, nature is assumed to be the only one factor affecting the realized return, i.e. high return $\left(R_{H}\right)$ or low return $\left(R_{L}\right)$. In period one, the probability of high return is $p$ (and $0<p<1$ ). However, under the assumption of state-dependence, the probability of high return occurring in period two will be contingent on the realized return in period one. If the realized return in period one is high (low), the probability of high return occurring in period two will be $p_{h}\left(p_{l}\right)$. It is assumed that $0<p_{l}<p<p_{h}<1$. While the realized return in either period one or period two is the manager's private information, the probabilities $p, p_{h}$ and $p_{l}$ can be estimated objectively according to the past operating results, and regarded as common information. In the paper, the principal will take the information into account while planning the related audit policy. Specifically, we incorporate the exogenous variables $p, p_{h}$ and $p_{l}$ into the principal's objective function to develop the optimal two-period audit policy. At the end of each period, the manager is required to declare a return level (either high return $\left(R_{H}\right)$ or low return $\left.\left(R_{L}\right)\right)$ to the principal, and transfers a portion $(\alpha)$ of the return to the latter. The transferring agreement brings about an incentive for the manager to under-declare the return.

This paper allows the principal to consider a two- period conditional audit mechanism to deter the manager's possible under-declaration of return. At the end of each period, the principal needs to determine whether to employ the auditor at cost $C$ to audit the low return declared by the manager. If the auditor finds the under-declaration of return, the manager will be required to pay a penalty of $\bar{P}$. Following the previous literature (e.g. Malik [11]), the penalty of $\bar{P}$ is set as a maximal possible amount to minimize the expected audit cost, and it can be regarded as a legally specified limit on liability. Under a conditional audit mechanism, the audit policy for the second period is likely to be dependent on the audit result in the first period. In period one, the probability to audit the low return declared by the manager is assumed to be $A$. However, if the under-declaration of return in period one is found and revealed by the auditor, the audit probability for the second period will be enhanced up to $A^{\prime}(\equiv A+a)$ provided the manager declares a low return once more in period two; otherwise, the audit probability will remain to be $A$, where $0 \leq A \leq 1,0 \leq a \leq 1-A$, and $A \leq A^{\prime}$. That is so-called "conditional audit" used in this paper.
Furthermore, the auditor's audit quality is defined as a probability, $r$ that the manager's under-declaration can be found by the auditor. In other words, there remains a probability, $1-r$ that the auditor will be unable to disclose the manager's dishonest behavior even if the former has accomplished the related audit task. In this paper, we exclude the possibility of blackmail or collusion between the auditor and the manager. Both $C$ and $r$ are assumed to be the common information of all parties involved. The following is the timing on the relevant events.

1) The principal and the manager achieve an agreement that the latter will transfer a certain portion $(\alpha)$ of the return to the former.

2) Nature determines the realized return in period one, which is a high return $\left(R_{H}\right)$ with the probability of $p$ or a low return $\left(R_{L}\right)$ with the probability of $1-p$.

$3)$ The manager declares the return in period one $\left(\hat{R}_{1}\right)$ to the principal and will transfer $\alpha \cdot \hat{R}_{1}$ to the latter.

4) The principal assigns the auditor to undertake audit task at $\operatorname{cost} C$ with an audit probability $A$ if the manager declares a low return in period one (i.e. $\hat{R}_{1}=R_{L}$ ).

5) The auditor presents an audit report. If the under-declaration of return is disclosed, the manager will have to pay the principal a penalty of $\bar{P}$, assumed to be larger than $\alpha \cdot\left(R_{H}-R_{L}\right)$ for compensation and punishment. Also, the manager's dishonest record will be kept for reference in next period.

6) Nature determines the realized return in period two once more. If the realized return in period one is $R_{H}\left(R_{L}\right)$, the probability of high return occurring in period two will be $p_{h}\left(p_{l}\right)$, where $p_{l}<p<p_{h}$.

7) The manager declares the return in period two, $\hat{R}_{2}$, and will transfer $\alpha \cdot \hat{R}_{2}$ to the principal.

8) The principal sends the auditor at $\operatorname{cost} C$ with a probability $A$ if the manager was not found under-declaring the return in period one and declares a low return in period two (i.e. $\hat{R}_{2}=R_{L}$ ), but with a probability $A^{\prime}$ if the manager was found under-declaring the return in period one and declares a low return in period two, where $A \leq A^{\prime}$.

9) The auditor presents an audit report, and the manager will be required to pay a penalty of $\bar{P}$ if the under-declaration of return is disclosed.

10) Transfer takes place.

Based on the self-interested and rational assumption, if the outcome in either period one or period two is a low realized return, the manager will necessarily choose to declare a low return to the principal. However, if the outcome is a high realized return, the manager will be likely to truthfully declare a high return or dishonestly declare a low return, depending on the result of cost and benefit analysis. In the first period, if the realized return is a high one, the manager will need to evaluate the dif- 
ference of transferring amounts $\left(\alpha\left(R_{H}-R_{L}\right)\right)$, the expected penalty $(A r \bar{P})$, and the unfavorable effect on the audit probability in period two (possibly adjusted from $A$ to $A^{\prime}$ ) in order to make an optimal declaring decision. In the second period, if the outcome is a high realized return, whether the manager chooses under-declaration or not will be contingent on the comparison between two factors, i.e. the benefit from the difference of transferring amounts $\left(\alpha\left(R_{H}-R_{L}\right)\right)$ and the loss from the expected penalty ( $A r \bar{P}$ or $A^{\prime} r \bar{P}$ depending on the previous audit result).

Since the realized return is the manager's private information, the principal's audit policy will be dependent on the return declared by the manager. On the basis of cost and benefit, the principal will take audit action only when the manager declares a low return. It is assumed that the auditor is required to present some evidence to support her audit report on the under-declared return, and the evidence cannot be falsified. Hence, the audit result will necessarily be a low return if the realized return is low, but if the realized return is high, the audit result will be subject to the effect of the audit quality $(r)$.

\section{The Analyses}

\subsection{The Agent's Strategy}

In this section, we first characterize the manager's possible strategies under the variety of parameter combinations. As the aforementioned, on the basis of the self-interested and rational assumption, the manager will consistently declare low return to the principal if the realized return in either period one or period two is low. Moreover, the factors influencing the manager's declaration behavior include the transferring ratio of return declared $(\alpha)$, the penalty $(\bar{P})$, the audit probabilities $(A$ and $\left.A^{\prime}\right)$ and the audit quality $(r)$. Using the relative relations among those parameters, we infer three possible strategies that the manager will take into account. They are shown in Lemmas 1 to 3, respectively. To simplify the denotation, we let $R_{H}-R_{L}$ be $\Delta R$ in the latter analyses, and $\alpha \Delta R$ denotes the manager's maximal possible benefit while choosing to under-declare the return.

\section{Lemma 1 :}

If $\alpha \Delta R \leq A r \bar{P}$, the manager will honestly declare the return in each period. That is, when the realized return in period one (or in period two) is high (i.e. $R_{1}=R_{H}$ or $R_{2}=R_{H}$ ), the manager will consistently declare high return to the principal (i.e. $\hat{R}_{1}=R_{1}=R_{H} \quad$ or $\hat{R}_{2}=R_{2}=R_{H}$ ).

\section{[Proof] See Appendix 1.}

\section{Lemma 2:}

Under the condition of $\operatorname{Ar} \bar{P}<\alpha \Delta R \leq A^{\prime} r \bar{P}$, when the realized return in period two is high (i.e. $R_{2}=R_{H}$ ), the manager will under-declare the return if she has no un- der-declaration record; but she will honestly declare high return if she was found under-declaring return in period one. Nevertheless, when the realized return in period one is high (i.e. $R_{1}=R_{H}$ ), the manager will choose to under-declare the return in period one.

[Proof] See Appendix 2.

\section{Lemma 3:}

Under the condition of $\alpha \Delta R>A^{\prime} r \bar{P}$, when the realized return in period one (or in period two) is high (i.e. $R_{1}=R_{H}$ or $\left.R_{2}=R_{H}\right)$, the manager will choose to under-declare the return to the principal (i.e. $\hat{R}_{1}=R_{L}<R_{1}$ or $\hat{R}_{2}=R_{L}<R_{2}$ ).

[Proof] See Appendix 3.

\subsection{The Principal's Strategy}

On the other hand, while facing the manager's possible strategy responses, the principal first needs to consider if it is economically desirable to use audit measure as an incentive mechanism. The precondition concerned is that the expected payoff from auditing should exceed the related cost (i.e. $r p \bar{P}>C$ ). Otherwise, the audit mechanism will not be conducted. Hence, we regard $r p \bar{P}>C$ as an implied assumption in the latter analysis.

To highlight the difference between $A$ and $A^{\prime}$ $(\equiv A+a)$ and search for the optimal value of $a$, we rewrite the preconditions in the Lemmas aforementioned as:

$$
\begin{gathered}
\alpha \Delta R \leq \operatorname{Ar} \bar{P} \Rightarrow 0<\alpha \Delta R / r \bar{P} \leq A \leq 1 \\
A r \bar{P}<\alpha \Delta R \leq(A+a) r \bar{P} \Rightarrow 0 \leq A<\alpha \Delta R / r \bar{P} \leq A+a \leq 1
\end{gathered}
$$

where $0 \leq a \leq 1-A$

$$
\alpha \Delta R>(A+a) r \bar{P} \Rightarrow 0 \leq A \leq A+a<\alpha \Delta R / r \bar{P}
$$

where $0 \leq A \leq 1$ and $0 \leq a \leq 1-A$.

Intuitively, if $\alpha \Delta R<r \bar{P}$ (or $\alpha \Delta R / r \bar{P}<1$ ), there can exist the Situations (1), (2) or (3). Specifically, the Situation (1) can be called as a "deterrent audit policy" since the audit policy will effectively deter the manager's under-declaring behavior. The Situation (2) can be referred to as a "punitive conditional audit policy" in that the audit probability in period two will be adjusted up to a deterrent (higher) audit probability from a normal (lower) audit probability if the manager was found under-declaring the return in period one. Finally, the Situation (3) can be regarded as a "laissez-faire audit policy," in which the manager will choose to under-declare the return due to no sufficient audit influence. It is assumed that the principal can obtain the expected payoffs $\pi_{1}, \pi_{2}$ and $\pi_{3}$ under Situations (1), (2) and (3), respectively. In Lemmas 4 and 5, it will be shown that the audit policy from situation (1) will dominate that from either situation (2) or Situation (3). Hence, provided $\alpha \Delta R<r \bar{P}$, the au- 
dit policy from situation (1) will be the principal's optimal strategy in light of maximizing her expected payoff.

\section{Lemma 4:}

If $\alpha \Delta R<r \bar{P}$, then the principal's maximal expected payoff in the Situation (1), $\pi_{1}^{*}$, will be larger than that in the Situation (2), $\pi_{2}^{*}$.

\section{[Proof] See Appendix 4.}

Lemma 4 indicates that, as the incentive from under-declaring is insignificant (i.e. $\alpha \Delta R<r \bar{P}$ ), the deterrent audit policy (from Situation (1)) will be better than the punitive conditional audit policy (from Situation (2)) such that the principal can obtain a larger expected payoff.

\section{Lemma 5:}

If $\alpha \Delta R<r \bar{P}$, then the principal's maximal expected payoff in the situation (1), $\pi_{1}^{*}$, will be larger than that in the Situation (3), $\pi_{3}^{*}$.

\section{[Proof] See Appendix 5.}

Similarly, as the incentive from under-declaring is insignificant, Lemma 5 points out that the deterrent audit policy (from Situation (1)) will dominate the laissez-faire audit policy (from Situation (3)) as well. Accordingly, we can infer the following result in Proposition 1.

\subsection{Deterrent Audit Policy}

\section{Proposition 1:}

If the benefit of under-declaring return is relatively insignificant (i.e. $\alpha \Delta R<r \bar{P}$ ), the principal's optimal audit policy will be conducting random audit with the audit probability of $A=\alpha \Delta R / r \bar{P}$, provided audit is cost-effective (i.e. $C<r p \bar{P}$ ), and $a$ will have no effect on the manager's declaration of the return in period two.

[Proof] By the Lemmas 4 and 5, it's straightforward that the principal can maximize the expected payoff by using the audit policy from the Situation (1) and achieve a maximal expected payoff, $\pi_{1}^{*}$.

As Proposition 1 shows, if the manager's benefit of under-declaring return is less than the expected penalty under complete audit (i.e. $\alpha \Delta R<r \bar{P}$ ), the optimal audit policy will be the deterrent audit policy (from Situation (1)). In that case, the principal will implement random audit action (i.e. $A=\alpha \Delta R / r \bar{P}$ ) to induce the manager to honestly declare her return, and to totally deter the under-declaring behavior. Obviously, since the manager necessarily chooses the honest declaration in period one, it is unnecessary to use a conditional audit probability in period two.

The deterrent audit policy may be not desirable ex post since audit is costly, but it reduces the expected audit cost and maximize the principal's expected payoff ex ante. Although it is doubtful that the principal would be able to adhere to her announced audit policy, the reputational forces could contribute toward inducing the principal to make such a commitment, especially in a repeated game setting such as royalty or tax collection. Hence, if the probability of random audit doesn't change dramatically over time, the manager could resort to inferences according to the principal's previous behavior.

As for when the manager's benefit of under-declaring return is not less than the expected penalty under complete audit (i.e. $\alpha \Delta R \geq r \bar{P}$ ), the optimal audit policy will need a different consideration. Firstly, we present a few technical definitions in the following Lemma 6.

\section{Lemma 6:}

Let

$$
\begin{aligned}
C_{1} & \equiv\left[r p \bar{P}+(1-p) r p_{l} \bar{P}+(1-r) r p p_{h} \bar{P}\right] /(2-r p), \\
C_{2} & \equiv\left[r p \bar{P}+(1-p) r p_{l} \bar{P}+(1+r) r p p_{h} \bar{P}\right] /(2+r p),
\end{aligned}
$$

$$
A_{1} \equiv\left[2\left(r^{2} p p_{h} \bar{P}-C\right)-r p C+r p \bar{P}+(1-p) r p_{l} \bar{P}+(1-r) r p p_{h} \bar{P}\right] /\left[2 r p\left(r p_{h} \bar{P}-C\right)\right]
$$

and

$$
a_{1} \equiv\left[(2-r p) C-r p \bar{P}-(1-p) r p_{l} \bar{P}-(1-r) r p p_{h} \bar{P}\right] /\left[2 r p\left(r p_{h} \bar{P}-C\right)\right]=1-A_{1}
$$

under $\alpha \Delta R \geq r \bar{P}$ and $p_{l}<p<p_{h}$, we have $C_{1}<C_{2}$. Also, if $C_{1}<C<C_{2}$, both $0<A_{1}<1$ and $0<a_{1}<1$ are assured.

\section{[Proof] See Appendix 6.}

\subsection{Conditional Audit Policy}

In Lemma 6, $C_{1}$ and $C_{2}$ are used to set up the triggering points for different audit policies in light of audit cost. Additionally, $A_{1}$ denotes a certain random audit probability, and $a_{1}$ is used as a punitive audit factor.
Using the related denotation, we characterize the principal's optimal audit policies in Proposition $2 a$.

Proposition 2a:

Following the denotation in Lemma 6, as the benefit of under-declaring return is sufficiently large (i.e. $\alpha \Delta R \geq r \bar{P})$ and there is a state-dependent return in period two (i.e. $p_{l}<p<p_{h}$ ), the principal's optimal audit policy will be conducting,

1) uniformly complete audit (i.e. $A=A^{\prime}=1$ and $a=0)$ if $C \leq C_{1}$;

2) some kind of conditional audit (i.e. $A=A_{1}, \quad a=a_{1}$, 
and $\left.A^{\prime}=A_{1}+a_{1}=1\right)$ if $C_{1}<C<C_{2}$, or;

3) no audit (i.e. $A=0$ ) if $C \geq C_{2}$.

[Proof] See Appendix 7.

Provided the benefit of under-declaring return is considerably significant (i.e. $\alpha \Delta R \geq r \bar{P}$ ), based on Lemma 3, the manager necessarily chooses to under-declare her return when a high return is realized. As Proposition $2 a$ shows, to respond to the manager's strategy, the principal will implement an uniformly complete audit policy (i.e. $A=A^{\prime}=1$ ) in each period if audit cost is relatively negligible (i.e. $C \leq C_{1}$ ). But if audit cost is relatively larger (i.e. $C \geq C_{2}$ ), no audit action (i.e. $A=0$ ) will be a desirable policy. In contrast, as the audit cost is moderate (i.e. $C_{1}<C<C_{2}$ ), the optimal policy will be a conditional audit policy (i.e. $A=A_{1}<1$ and $A^{\prime}=1>A$ ). In this case, the principal will first take a random audit action in period one, and then decide whether to adjust up the audit probability in period two, depending on the audit result in previous period.

Since $a_{1}\left(=1-A_{1}\right)$ corresponds to the punitive effect of conditional audit policy, a larger $a_{1}$ implies the principal can conduct a random audit in period one with a lower audit probability, and then implement a complete audit in period two when the manager has a under-reporting record and declares a low return once more. In Proposition $2 b$, we examine the impacts of audit cost, audit quality, and under-reporting penalty on the punitive effect of conditional audit policy.

Proposition $2 b$ :

As conditional audit policy is the principal's optimal choice, ceteris paribus, an increase in audit cost results in a larger punitive effect of conditional audit (i.e. $\left.\partial a_{1} / \partial C>0\right)$. However, a rise in audit quality or penalty results in a decrease in the punitive effect of conditional audit (i.e. $\partial a_{1} / \partial r<0$ and $\left.\partial a_{1} / \partial \bar{P}<0\right)$ ). The former leads to a lower random audit probability in period one ( $A_{1}$ ), but the latter leads to a higher one (since $\left.A_{1}=1-a_{1}\right)$.

[Proof] See Appendix 8.

As conditional audit policy is an optimal one, an increase in audit cost will be more unfavorable to audit action and result in a lower random audit probability in period one (a more punitive effect). Conversely, as audit quality or penalty grows, the expected benefit of random audit in period one will increase, and it leads to a higher random audit probability in period one (a less punitive effect). In Proposition $2 c$, we further investigate how the degree of return dependence in two consecutive periods influences the punitive effect of conditional audit policy.

Proposition 2c:

Let $\Delta p_{h} \equiv p_{h}-p$ and $\Delta p_{l} \equiv p-p_{l}$. Following the preconditions in Proposition $2 b$, if the realization of high return in period one brings about a much more chance of realizing a high return in period two (i.e. a larger $\Delta p_{h}$ ), the principal will reduce the punitive effect in conditional audit policy (i.e. a smaller $a_{1}$ ). Nevertheless, if the realization of low return in period one results in a much more chance of realizing a low return in period two or a much less chance of realizing a high return in period two (i.e. a larger $\Delta p_{l}$ ), the principal will enlarge the punitive effect in conditional audit policy (i.e. a larger $a_{1}$ ). The former leads to a higher random audit probability in period one $\left(A_{1}\right)$, but the latter leads to a lower one (since $\left.A_{1}=1-a_{1}\right)$.

[Proof] See Appendix 9.

As Proposition $2 c$ shows, $\Delta p_{h}$ and $\Delta p_{l}$ have opposite impacts on the punitive effect of conditional audit policy. Intuitively, since $\Delta p_{h}$ grows means that a high return in period one will lead to a much more chance of realizing a high return in period two, it become more favorable to increase the frequency of audit in period one and results in a higher random audit probability in the period (a less punitive effect). In contrast, if a low return in period one leads to a much less chance of realizing a high return in period two, it will be less favorable to enlarge the audit in period one and results in a lower random audit probability in the period (a more punitive effect).

\subsection{No Conditional Audit Scenario}

Finally, we are interested in how the optimal audit policy will be if the return-dependence in two periods vanishes totally. Is there any possibility for conditional audit policy to be desirable? As shown in the following proposition, the answer seems negative in light of the assumptions in the paper.

Proposition 3:

Using the denotation in Proposition 2a, if the benefit of under-declaring return is relatively significant (i.e. $\alpha \Delta R \geq r \bar{P}$ ), and the return in period two is independent of that in period one (i.e. $p_{l}=p=p_{h}$ ), it will be not desirable for the principal to conduct any conditional audit, and the optimal audit strategy will be either

1) complete audit policy (i.e. $A=A^{\prime}=1$ and $a=0$ ) provided $C \leq r p \bar{P}$, or

2) no audit policy (i.e. $A=0$ ) provided $C>r p \bar{P}$.

[Proof] Following the proof of Proposition $2 a$, under $p_{l}=p=p_{h}$, both $C_{1}$ and $C_{2}$ converge to $r p \bar{P}$, i.e. $C_{1}=C_{2}=r p \bar{P}$. Hence, the result (2) in Proposition $2 a$ will vanish.

In Proposition 3, we let both $p_{l}$ and $p_{h}$ converge to $p$ so that the returns in periods one and two are independent each other. In the specific case, we obtain a result that a conditional audit policy is uneconomical and inadequate if the realized return in period one has no effect on the realization of return in period two. As a result, the principal will choose either a complete-audit policy or a no-audit policy, depending on the size of au- 
dit cost (i.e. $C \leq r p \bar{P}$ or $C>r p \bar{P}$ ).

\section{Conclusion}

In a state-dependent audit situation, we find that the principal's optimal audit policy will be a deterrent audit policy if the manager's benefit of under-declaring return is less than the expected penalty under complete audit and audit cost is insignificant. Nevertheless, if the benefit of under-declaring return is considerably significant, and the manager necessarily chooses to under-declare the return, the principal's optimal audit policy will be dependent on audit cost. Ceteris paribus, if audit cost is relatively negligible, the principal will take a uniformly complete audit policy; but if audit cost is relatively larger, the principal will tend to totally abandon audit action in consideration of costs and benefits related. More interestingly, as the audit cost is moderate, we find that a conditional audit policy will be an optimal choice. In that case, while the principal's audit action cannot deter the manager's under-declaring behavior, the conditional audit policy indeed can make audit action more efficient and cost-effective.

\section{REFERENCES}

[1] R. Antle, "The Auditor as an Economic Agent," Journal of Accounting Research, Vol. 20, No. 2, 1982, pp. 503527. doi:10.2307/2490884

[2] D. P. Baron and D. Besanko, "Regulation, Asymmetric Information and Auditing," RAND Journal of Economics, Vol. 15, No. 4, 1984, pp. 447-470. doi: $10.2307 / 2555518$

[3] J. S. Demski and D. E. M. Sappington, "Hierarchical
Regulatory Control," RAND Journal of Economics, Vol. 18, No. 3, 1987, pp. 369-383. doi:10.2307/2555602

[4] S. Baiman, J. H. Evans III and J. Noel, "Optimal Contracts with a Utility-Maximizing Auditor," Journal of Accounting Research, Vol. 25, No. 2, 1987, pp. 217-244. doi: $10.2307 / 2491016$

[5] E. Helland, "The Enforcement of Pollution Control Laws: Inspections, Violations, and Self-Reporting," Review of Economics and Statistics, Vol. 80, No. 1, 1998, pp. 141153. doi:10.1162/003465398557249

[6] M. Landsberger and I. Meilijson, "Incentive Generating State Dependent Penalty System: The Case of Income Tax Evasion," Journal of Public Economics, Vol. 19, No. 3, 1982, pp. 333-352. doi:10.1016/0047-2727(82)90060-3

[7] J. Greenberg, “Avoiding Tax Avoidance: A (Repeated) Game-Theoretic Approach," Journal of Economic Theory, Vol. 32, No. 1, 1984, pp. 1-13. doi:10.1016/0022-0531(84)90071-1

[8] W. Harrington, "Enforcement Leverage When Penalties Are Restricted," Journal of Public Economics, Vol. 37, No, 1, 1988, pp. 29-53. doi:10.1016/0047-2727(88)90003-5

[9] L. Friesen, "Targeting Enforcement to Improve Compliance with Environmental Regulations," Journal of Environmental Economics and Management, Vol. 46, No. 1, 2003, pp. 72-85. doi:10.1016/S0095-0696(02)00033-5

[10] S. L. Stafford, "Self-Policing in a Targeted Enforcement Regime," Southern Economic Journal, Vol. 74, No. 4, 2008, pp. 934-951.

[11] A. S. Malik, "Self-Reporting and the Design of Policies for Regulating Stochastic Pollution," Journal of Environmental Economics and Management, Vol. 24, No. 3, 1993, pp. 241-257. doi:10.1006/jeem.1993.1016 


\section{Appendix 1 (Proof of Lemma 1)}

Under $\quad \alpha \Delta R \leq A r \bar{P}\left(\leq A^{\prime} r \bar{P}\right)$, if $R_{2}=R_{H}$, then $\hat{R}_{2}=R_{H}$ since the expected penalty will be too large for the manager to under-declare the return. By the same token, if $R_{1}=R_{H}$, then $\hat{R}_{1}=R_{H}$ since any audit result in period one will not affect her declaration decision as well as expected payoff in period two, and

$$
\begin{aligned}
\mathrm{E} & \left(\hat{R}_{1}=R_{H} \mid R_{1}=R_{H}\right)-\mathrm{E}\left(\hat{R}_{1}=R_{L} \mid R_{1}=R_{H}\right) \\
= & \left(R_{H}-\alpha R_{H}\right)+p_{h}\left(R_{H}-\alpha R_{H}\right)+\left(1-p_{h}\right)\left(R_{L}-\alpha R_{L}\right)-\left(R_{H}-\alpha R_{L}-A r \bar{P}\right) \\
& -A r\left[p_{h}\left(R_{H}-\alpha R_{H}\right)+\left(1-p_{h}\right)\left(R_{L:}-\alpha R_{L}\right)\right]-(1-A r)\left[p_{h}\left(R_{H}-\alpha R_{H}\right)+\left(1-p_{h}\right)\left(R_{L:}-\alpha R_{L}\right)\right] \\
= & \alpha R_{L}-\alpha R_{H}+A r \bar{P}=-\alpha \Delta R+A r \bar{P} \geq 0(\text { if } \alpha \Delta R \leq A r \bar{P})
\end{aligned}
$$

\section{Appendix 2 (Proof of Lemma 2)}

If the realized return in period two is $R_{H}$, under the condition of $\alpha \Delta R>A r \bar{P}$, the manager will choose to under-declare the return if she has a clean record; however, she won't do that if she was found under-declaring return in period one since $\alpha \Delta R \leq A^{\prime} r \bar{P}$.

On the other hand, if the realized return in period one is $R_{H}$, the manager will be inclined to under-declare the return since

$$
\begin{aligned}
& \mathrm{E}\left(\hat{R}_{1}=R_{H} \mid R_{1}=R_{H}\right)-\mathrm{E}\left(\hat{R}_{1}=R_{L} \mid R_{1}=R_{H}\right)=\alpha R_{L}-\alpha R_{H}+\operatorname{Ar} \bar{P}+\operatorname{Arp}_{h}\left(\alpha R_{H}-\alpha R_{L}-A r \bar{P}\right) \\
& =-\alpha \Delta R+\operatorname{Ar} \bar{P}+\operatorname{Arp}_{h}(\alpha \Delta R-A r \bar{P})=(\alpha \Delta R-A r \bar{P})\left(\operatorname{Arp}_{h}-1\right)<0\left(\because \alpha \Delta R>A r \bar{P} \text { and } \operatorname{Arp}_{h}<1\right)
\end{aligned} .
$$

\section{Appendix 3 (Proof of Lemma 3)}

Under the condition of $\alpha \Delta R>A^{\prime} r \bar{P}(\geq A r \bar{P})$, if the realized return in period two is high (i.e. $R_{2}=R_{H}$ ), the manager will be inclined to under-declare the return whether she was found under-declaring return in period one or not. Meanwhile, if the realized return in period one is $R_{H}$, the manager will be also inclined to under-declare the return since

$$
\begin{aligned}
\mathrm{E} & \left(\hat{R}_{1}=R_{H} \mid R_{1}=R_{H}\right)-\mathrm{E}\left(\hat{R}_{1}=R_{L} \mid R_{1}=R_{H}\right) \\
= & \left(R_{H}-\alpha R_{H}\right)-\left(R_{H}-\alpha R_{L}-\operatorname{Ar} \bar{P}\right) \\
& +p_{h}\left(R_{H}-\alpha R_{L}-\operatorname{Ar} \bar{P}\right)-\operatorname{Arp}\left[R_{H}-\alpha R_{L}-(A+a) r \bar{P}\right] \\
& -p_{h}\left(R_{H}-\alpha R_{L}-\operatorname{Ar} \bar{P}\right)+\operatorname{Arp}_{h}\left(R_{H}-\alpha R_{L}-\operatorname{Ar} \bar{P}\right) \\
= & \alpha R_{L}-\alpha R_{H}+\operatorname{Ar} \bar{P}-\operatorname{Arp}_{h}[\operatorname{Ar} \bar{P}-(A+a) r \bar{P}] \\
= & -\alpha \Delta R+\operatorname{Ar} \bar{P}+\operatorname{Arp}_{h} \cdot \operatorname{ar} \bar{P} \\
& <0\left(\because \alpha \Delta R \geq(A+a) r \bar{P}>\operatorname{Ar} \bar{P}+\left(\operatorname{Arp}_{h}\right) \operatorname{ar} \bar{P}\right)
\end{aligned}
$$

\section{Appendix 4 (Proof of Lemma 4)}

In Situation (1), the principal's expected payoff is

$$
\begin{aligned}
\pi_{1}= & p\left[\alpha R_{H}+p_{h} \alpha R_{H}+\left(1-p_{h}\right)\left(\alpha R_{L}-A C\right)\right] \\
& +(1-p)\left[\alpha R_{L}-A C+p_{l} \alpha R_{H}\right. \\
& \left.+\left(1-p_{l}\right)\left(\alpha R_{L}-A C\right)\right]
\end{aligned}
$$

To maximize $\pi_{1}$, subject to $A \geq \alpha \Delta R / r \bar{P}(>0)$, $A+a \leq 1$ and $a \geq 0$, we find the only optimal combination of $A^{*}=\alpha \Delta R / r \bar{P}$ and $0 \leq a^{*} \leq 1-(\alpha \Delta R / r \bar{P})$, satisfying all of the Kuhn-Tucker conditions. Actually, the $a^{*}$ will never be used in that case since the manager will honestly declare her return in period one under $A^{*}=\alpha \Delta R / r \bar{P} \quad(\equiv \underline{A})$. Thus we get the maximal expected payoff

$$
\begin{aligned}
\pi_{1}^{*}= & 2 \alpha R_{L}+p\left(1+p_{h}\right) \alpha \Delta R+(1-p) p_{l} \alpha \Delta R \\
& -\left(2-p-p_{l}-p p_{h}+p p_{l}\right) \alpha \Delta R C / r \bar{P} .
\end{aligned}
$$

On the other hand, in Situation (2), the principal's expected payoff is 


$$
\begin{aligned}
\pi_{2}= & p\left\{\alpha R_{L}+A(r \bar{P}-C)+p_{h}\left\{A r \alpha R_{H}+(1-A r)\left[\alpha R_{L}+A(r \bar{P}-C)\right]\right\}\right. \\
& \left.+\left(1-p_{h}\right)\left\{A r\left[\alpha R_{L}-(A+a) C\right]+(1-A r)\left(\alpha R_{L}-A C\right)\right\}\right\} \\
& +(1-p)\left\{\alpha R_{L}-A C+p_{l}\left[\alpha R_{L}+A(r \bar{P}-C)\right]+\left(1-p_{l}\right)\left(\alpha R_{L}-A C\right)\right\} \\
= & 2 \alpha R_{L}+p p_{h} A r \alpha \Delta R-2 A C+p p_{h} A r A C-p\left(1-p_{h}\right) A r a C \\
& +\left[p p_{h}(1-A r) A+(1-p) p_{l} A+p A\right] r \bar{P}
\end{aligned}
$$

To maximize $\pi_{2}$, subject to $0 \leq A<\alpha \Delta R / r \bar{P}$, $\alpha \Delta R / r \bar{P} \leq A+a \leq 1$ and $a \geq 0$, it is derived that only the combination of $A^{*}=\alpha \Delta R / r \bar{P}-\varepsilon \quad(\equiv \underline{A}-\varepsilon)$ and $a^{*}=\varepsilon$ (where $\varepsilon>0$ and $\varepsilon>0$ ) can satisfy all of the Kuhn-Tucker conditions. Hence, the maximal expected payoff becomes

$$
\begin{aligned}
\pi_{2}^{*}= & 2 \alpha R_{L}+p p_{h}(\underline{A}-\varepsilon) r \alpha \Delta R-2(\underline{A}-\varepsilon) C+p p_{h}(\underline{A}-\varepsilon)^{2} r C-p\left(1-p_{h}\right)(\underline{A}-\varepsilon) r a C \\
& +\left[p p_{h}(1-(\underline{A}-\varepsilon) r)(\underline{A}-\varepsilon)+(1-p) p_{l}(\underline{A}-\varepsilon)+p(\underline{A}-\varepsilon)\right] r \bar{P}
\end{aligned}
$$
have

Hence, overlooking the terms involved with $\varepsilon$ we

$$
\pi_{1}^{*}-\pi_{2}^{*} \cong p p_{h} \underline{A} C(1-\underline{A} r)+\left[p\left(1-p_{l}\right)+p_{l}\right] \underline{A} C>0,
$$

where $0<\underline{A}=\alpha \Delta R / r \bar{P}<1$.

\section{Appendix 5 (Proof of Lemma 5)}

In Situation (3), the principal's expected payoff is

$$
\begin{aligned}
\pi_{3}= & 2 \alpha R_{L}-2 A C-p \operatorname{Ara} C+p A r \bar{P} \\
& +p p_{h} \operatorname{Ar} \bar{P}+(1-p) p_{l} \operatorname{Ar} \bar{P}+p p_{h} \operatorname{Arar} \bar{P}
\end{aligned} .
$$

To maximize $\pi_{3}$, subject to $A+a<\alpha \Delta R / r \bar{P}, A \geq 0$ and $a \geq 0$, we find that only the optimal combination of $A^{*}=\alpha \Delta R / r \bar{P}-\varepsilon \quad($ where $\varepsilon>0$ and $\varepsilon \rightarrow 0$ ) and $a^{*}=0$ can satisfy all of the Kuhn-Tucker conditions. Hence, the maximal expected payoff become

$$
\pi_{3}^{*}=2 \alpha R_{L}-2 A C+\left(p+p p_{h}+p_{l}-p p_{l}\right)(\underline{A}-\varepsilon) r \bar{P},
$$

where $\underline{A} \equiv \alpha \Delta R / r \bar{P}$.

According to the proof of lemma 4 ,

$$
\begin{aligned}
\pi_{1}^{*}= & 2 \alpha R_{L}+p\left(1+p_{h}\right) \underline{A} r \bar{P}+(1-p) p_{l} \underline{A r} \bar{P} \\
& -\left(2-p-p_{l}-p p_{h}+p p_{l}\right) \underline{A C} .
\end{aligned}
$$

Hence, overlooking the terms involved with $\varepsilon$,

$$
\pi_{1}^{*}-\pi_{3}^{*} \cong\left[p p_{h}+p\left(1-p_{l}\right)+p_{l}\right] \underline{A} C>0,
$$

where $0<\underline{A}=\alpha \Delta R / r \bar{P}<1$.

\section{Appendix 6 (Proof of Lemma 6)}

Since

$$
\begin{aligned}
C_{1}< & C_{2} \\
\Leftrightarrow & (2+r p)\left[r p \bar{P}+(1-p) r p_{l} \bar{P}+(1-r) r p p_{h} \bar{P}\right] \\
& <(2-r p)\left[r p \bar{P}+(1-p) r p_{l} \bar{P}+(1+r) r p p_{h} \bar{P}\right] \\
\Leftrightarrow & r p \bar{P}+r p p_{h} \bar{P}+(1-p) r p_{l} \bar{P}<2 r p_{h} \bar{P} \\
\Leftrightarrow & p+p p_{h}+(1-p) p_{l}<2 p_{h} \\
\Leftrightarrow & \left(p_{h}-p\right)+\left[p_{h}-p p_{h}-(1-p) p_{l}\right] \\
& >0\left(\because p_{l}<p<p_{h}\right) \\
C_{1}< & C_{2} \text { holds under } \alpha \Delta R \geq r \bar{P} . \\
\text { On the other hand, } & A_{1}>0 \\
\Leftrightarrow & 2\left(r^{2} p p_{h} \bar{P}-C\right)-r p C+r p \bar{P}+(1-p) r p_{l} \bar{P} \\
& +(1-r) r p p_{h} \bar{P}>0\left(\because r p_{h} \bar{P}>C\right) \\
\Leftrightarrow & r p \bar{P}+(1-p) r p_{l} \bar{P}+(1+r) r p p_{h} \bar{P} \\
& >(2+r p) C \Leftrightarrow C<C_{2},
\end{aligned}
$$

and

$$
\begin{aligned}
& A_{1}<1 \\
& \Leftrightarrow 2\left(r^{2} p p_{h} \bar{P}-C\right)-r p C+r p \bar{P}+(1-p) r p_{l} \bar{P} \\
&+(1-r) r p p_{h} \bar{P}<2 r p\left(r p_{h} \bar{P}-C\right) \\
& \Leftrightarrow r p \bar{P}+(1-p) r p_{l} \bar{P}+(1-r) r p p_{h} \bar{P}<(2-r p) C \\
& \Leftrightarrow C>C_{1}
\end{aligned}
$$

Hence, $0<A_{1}<1$ if $C_{1}<C<C_{2}$.

Meanwhile, 


$$
\begin{aligned}
& a_{1}>0 \\
& \Leftrightarrow(2-r p) C-r p \bar{P}-(1-p) r p_{l} \bar{P}-(1-r) r p p_{h} \bar{P}>0 \\
& \Leftrightarrow(2-r p) C>r p \bar{P}+(1-p) r p_{l} \bar{P}+(1-r) r p p_{h} \bar{P} \\
& \Leftrightarrow C>C_{1},
\end{aligned}
$$

and

$$
\begin{aligned}
a_{1} & <1 \\
\Leftrightarrow & (2-r p) C-r p \bar{P}-(1-p) r p_{l} \bar{P}-(1-r) r p p_{h} \bar{P} \\
& <2 r p\left(r p_{h} \bar{P}-C\right) \\
\Leftrightarrow & (2+r p) C<r p \bar{P}+(1-p) r p_{l} \bar{P}+(1+r) r p p_{h} \bar{P} \\
\Leftrightarrow & C<C_{2}
\end{aligned}
$$

Hence, $0<a_{1}<1$ if $C_{1}<C<C_{2}$.

\section{Appendix 7 (Proof of proposition 2a)}

According to the result of Lemma 6 , as $\alpha \Delta R \geq r \bar{P}$, the principal's expected payoff will be

$$
\begin{aligned}
& \pi=2 \alpha R_{L}+\operatorname{Arp}_{\bar{P}}-2 A C-\operatorname{Aarp}+\operatorname{Arpp}_{h} \bar{P} \\
& +\operatorname{Aar}^{2} p p_{h} \bar{P}+\operatorname{Ar}(1-p) p_{l} \bar{P}
\end{aligned}
$$

To maximize $\pi$, subject to $A+a \leq 1, A \geq 0$, and $a \geq 0$, we find the following results. Firstly, as $C_{1}<C<C_{2}$, only the optimal combination of $A^{*}=A_{1}$, $a^{*}=a_{1}$, and $A^{\prime *}=A_{1}+a_{1}=1$ can satisfy all of the Kuhn-Tucker conditions. In that case, $0<A_{1}<1$ and $0<a_{1}<1$ will also be assured by Lemma 6 (since $\left.C_{1}<C<C_{2}\right)$. Secondly, as $C \leq C_{1}$, the combination of $A^{*}=1$ and $a^{*}=0$ (i.e. $A^{\prime *}=1$ ) will be the optimal solution, satisfying all of the Kuhn-Tucker conditions; but if $C \geq C_{2}$, the optimal solution will be $A^{*}=0$ (and $a$ has no effect).

\section{Appendix 8 (Proof of proposition 2b)}

$$
\begin{aligned}
& \partial a_{1} / \partial C \\
& =2 r p\left\{\left(r p_{h} \bar{P}-C\right)(2-r p)+\left[(2-r p) C-r p \bar{P}-(1-p) r p_{l} \bar{P}-(1-r) r p p_{h} \bar{P}\right]\right\} /\left[4 r^{2} p^{2}\left(r p_{h} \bar{P}-C\right)^{2}\right]>0\left(\text { if } C>C_{1}\right) \\
& \partial a_{1} / \partial r=-\left\{2 r p\left(r p_{h} \bar{P}-C\right)\left[p C+(1-p) p_{l} \bar{P}+\left(1-r p_{h}\right) p \bar{P}+(1-r) p p_{h} \bar{P}\right]\right. \\
& \left.+2 p\left(2 r p_{h} \bar{P}-C\right)\left[(2-r p) C-r p \bar{P}-(1-p) r p_{l} \bar{P}-(1-r) r p p_{h} \bar{P}\right]\right\} /\left[4 r^{2} p^{2}\left(r p_{h} \bar{P}-C\right)^{2}\right]<0\left(\text { if } C>C_{1}\right) \\
& \partial a_{1} / \partial \bar{P}=-\left\{2 r p\left(r p_{h} \bar{P}-C\right)\left[\left(1-(1-r) p_{h}\right) r p+(1-p) r p_{l}\right]\right. \\
& \left.+2 r^{2} p p_{h}\left[(2-r p) C-r p \bar{P}-(1-p) r p_{l} \bar{P}-(1-r) r p p_{h} \bar{P}\right]\right\} /\left[4 r^{2} p^{2}\left(r p_{h} \bar{P}-C\right)^{2}\right]<0\left(\text { if } C>C_{1}\right) .
\end{aligned}
$$

\section{Appendix 9 (Proof of proposition 2c)}

$$
\begin{aligned}
& \partial a_{1} / \partial \Delta p_{h}=-\left\{2 r^{2} p^{2} \bar{P}(1-r)\left(r p_{h} \bar{P}-C\right)+2 r^{2} p \bar{P}[(2-r p) C-r p \bar{P}\right. \\
& \left.\left.-(1-p) r p_{l} \bar{P}-(1-r) r p p_{h} \bar{P}\right]\right\} /\left[4 r^{2} p^{2}\left(r p_{h} \bar{P}-C\right)^{2}\right]<0\left(\text { if } C>C_{1}\right) \\
& \partial a_{1} / \partial \Delta p_{l}=r \bar{P}(1-p) /\left[2 r p\left(r p_{h} \bar{P}-C\right)\right]>0 .
\end{aligned}
$$

